\title{
Towards a versatile point-of-care system combining femtosecond laser generated microfluidic channels and direct laser written microneedle arrays
}

\author{
Anika Trautmann ${ }^{1}$, Gian-Luca Roth ${ }^{1}$, Benedikt Nujiqi ${ }^{1}$, Thomas Walther ${ }^{2}$ and Ralf Hellmann ${ }^{1}$
}

\begin{abstract}
Microneedle-based microfluidic systems have a great potential to become well-accepted medical devices for simple, accurate, and painless drug delivery and lab-on-a-chip diagnostics. In this work, we report on a novel hybrid approach combining femtosecond direct laser written microneedles with femtosecond laser generated microfluidic channels providing an important step towards versatile medical point-of-care systems. Hollow microneedle arrays are fabricated by a laser system designed for two-photon polymerization applications. Compression tests of two different types of truncated cone-shaped microneedle arrays prepared from OrmoComp ${ }^{\circledR}$ give information about the microneedle mechanical strength, and the results are compared to skin insertion forces. Three-dimensional microchannels are directly created inside PMMA bulk material by an ultrashort pulse laser system with vertical channels having adjustable cross-sectional areas, which allow attaching of microneedles to the microfluidic system. A comprehensive parameter study varying pulse duration and repetition rate is performed on two-photon polymerization to identify an optimal laser power range for fabricating microneedles using the same pulse duration and repetition rate as for microchannels. This addresses the advantage of a single laser system process that overcomes complex fabrication methods. A proof of concept flow test with a rhodamine B dye solution in distilled water demonstrates that the combination of microneedles and microchannels qualifies for microfluidic injection and extraction applications.
\end{abstract}

\section{Introduction}

Point-of-care solutions based on microneedles attract interest due to their advantages in handling, patient protection, and patient care compared to conventional systems. They offer an alternative to subcutaneous medication, which, for example, is currently the preferred insulin administration method in the treatment of diabetes $^{1,2}$. Furthermore, intradermal microneedle patches are a promising candidate to revolutionize vaccination underlining the constant demand for influenza

\footnotetext{
Correspondence: Anika Trautmann (trautmann.anika@gmail.com) ${ }^{1}$ Applied Laser and Photonics Group, University of Applied Sciences Aschaffenburg, Wuerzburger Strasse 45, 63743 Aschaffenburg, Germany ${ }^{2}$ Institute of Applied Physics, Technische Universität Darmstadt, Schlossgartenstrasse 7, 64289 Darmstadt, Germany
}

vaccinations $^{2,3}$ and research in cancer therapy ${ }^{4,5}$. Microneedle systems connected to fluidic microchannels even allow fluid extraction and meet the needs for fast and simple monitoring of physiological systems ${ }^{6,7}$. For all these applications, microneedle-based solutions are minimally invasive, injectable without medical experts, and minimize the risk of inflammation and infection ${ }^{1,8}$. Patients, especially children ${ }^{5,9}$, benefit from such painless ${ }^{10}$ and easy to handle systems improving medical care $^{3,11}$.

Several application types of microneedles ${ }^{12}$ have been realized with different fabrication techniques until now. Solid microneedle ${ }^{13}$, coated microneedle ${ }^{14}$, and dissolving microneedle $\mathrm{e}^{15,16}$ designs are distinguished to release drugs from a patch after perforating the skin or 
directly from the microneedle coating or dissolving microneedle into the skin. Moreover, hollow microneedles enable both drug injection ${ }^{3}$ and fluid extraction $^{6}$. A cone shape $e^{6,16,17}$ or pyramid shape $e^{7,18}$ often serves as a basis for this needle type. Needle designs with offset bores prevent clogging after insertion into the $\operatorname{skin}^{19}$, and imitating mosquito's fascicle is an option for optimizing the microneedle shape and reducing the insertion force $\mathrm{e}^{20,21}$. A favorable method to fabricate these microneedles is direct laser writing via twophoton absorption ${ }^{17}$. In contrast to other fabrication techniques like etching methods ${ }^{6,22}$ or microinjection molding ${ }^{23,24}$, three-dimensional microneedles with arbitrary shape and high resolution can be achieved in a single-step process. This is due to the fact that photosensitive material is only modified around the focus position of the applied laser beam. OrmoComp (micro resist technology, Berlin, Germany), an organicinorganic hybrid polymer, is an appropriate material to write microneedles with this nonlinear polymerization process showing enough hardness to penetrate the stratum corneum layer ${ }^{17}$ and being tested for biocompatibility ${ }^{25,26}$.

For accurate drug delivery and lab-on-a-chip diagnostics, microneedle arrays are combined with an additional microfluidic system. The fabrication of these systems is usually laborious and requires multiple processing steps (i.e., etching and bonding steps) ${ }^{7,27-29}$. Alternatively, some groups realized less complex openchannel approaches ${ }^{23,30}$. In this work, a femtosecond laser-based process is chosen to directly generate threedimensional microfluidic channels inside bulk PMMA chips $^{31}$. The idea of combining two-photon polymerization and subtractive femtosecond laser-based microfabrication is known from literature. It was used to realize microfluidic devices by ablating microgrooves in glass slabs, polymerizing microfilters inside them, and sealing the whole chip with cover glass with a single laser source $^{32}$ or to realize micromechanical sensors by illuminating glass, etching, and two-photon polymerization ${ }^{33}$. To emphasize the difference to our approach, it is worth to stress that etching is a time-consuming process taking $6 \mathrm{~h}$ in Tičkunas et $\mathrm{al}^{33}$ and that welding is a delicate procedure $^{32}$. Furthermore, both approaches require three main processes (ablation, polymerization, and welding or illumination, etching, and polymerization) to generate the presented devices. In notable contrast, we introduce a new approach with two main processes, microchannel creation and microneedle polymerization, in a hybrid process using only polymers and avoiding brittle ${ }^{18}$ and more expensive $^{34}$ glass for the microfluidic system.

Regions of PMMA are laser modified under ambient conditions and subsequently heated to create an internal three-dimensional microfluidic system with horizontal and vertical channels. After preparation, two-photon polymerized microneedles are placed on the open vertical channels to create a combined system of microchannels and microneedles. Fluidic functionality is demonstrated by a flow test with a dye rhodamine B solution in distilled water. We highlight the joint fabrication of microneedles and microchannels with a single laser system obtained by studying direct writing for different laser parameters.

\section{Results}

Microneedles with arbitrary shape are achieved by direct laser writing via two-photon polymerization. Besides cone-shaped designs, pyramid shapes belong to the possible needle geometries. The CAD design and scanning electron microscope (SEM) images of a pyramid shape with two offset bores is shown in Fig. 1a-c. This design is more complex than common cone-shaped designs and leads to a production time of $10 \mathrm{~min}$ per needle. Compared to a 10 times faster fabrication of truncated cone-shaped microneedles (Fig. 1e and f) using a helix design as schematically shown in Fig. 1d, this is significantly slower.

\section{Mechanical compression tests}

For two types of truncated cone-shaped microneedles on glass coverslips with laser-drilled holes, a compression test is performed. Figure 2a presents a detailed view of an array with the first type, thin microneedles, and Fig. $2 \mathrm{~b}$ a detailed view of an array with the second type, thick microneedles, taken with a SEM. The wall angle ${ }^{35}$ of the microneedles is calculated by measuring the needles height, tip radius, and base radius and is $11^{\circ}$ for thin microneedles and $24^{\circ}$ for thick microneedles. Both needle types show a reproducible fabrication process and an exact positioning above the laser-drilled holes covered by microneedles. In each case, eight specimens are examined by a compression test. Figure $2 \mathrm{c}$ and $\mathrm{d}$ present the test setup with a pressure pad lowering down onto thin (Fig. 2c) and thick (Fig. 2d) microneedle arrays positioned above laser-drilled holes on a glass coverslip. The force and displacement of the pressure pad lowered down onto the microneedle arrays is measured and the calculated force per needle is provided in Fig. 2e. The curves start with a constant force related to the distance until the pressure pad reaches the top of the microneedles. Then, the curves increase linearly, which displays elastic deformation of the needles. After a maximum, the curves decrease again and the deviation between curves of the same type increases. The maxima of the thin needle type $(7.3-9.9 \mathrm{mN})$ are smaller than the maxima of the thick needle type $(17.7-25.3 \mathrm{mN})$. This points to a higher stability of the thicker microneedles. Furthermore, the maximum is a transition point from elastic to plastic 
a

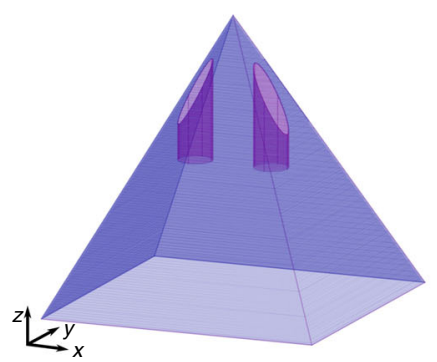

d

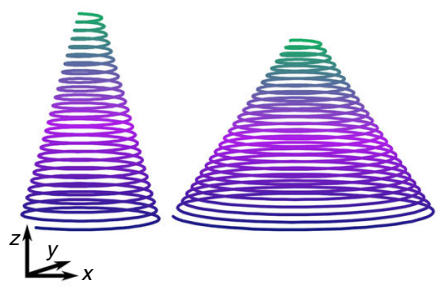

b

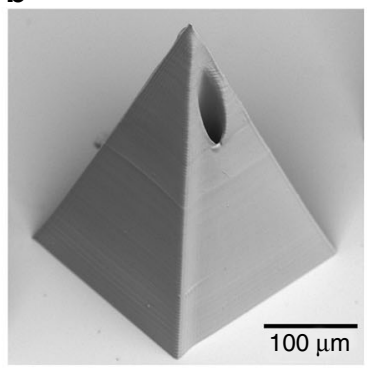

e

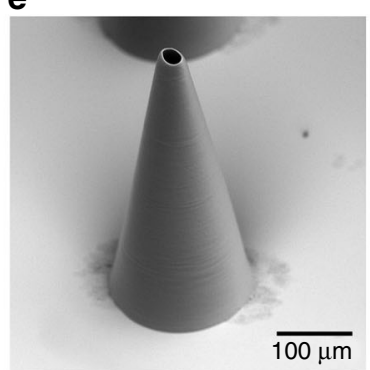

C

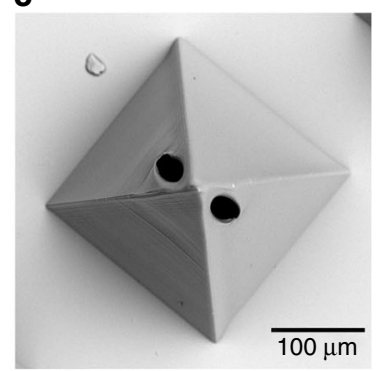

f

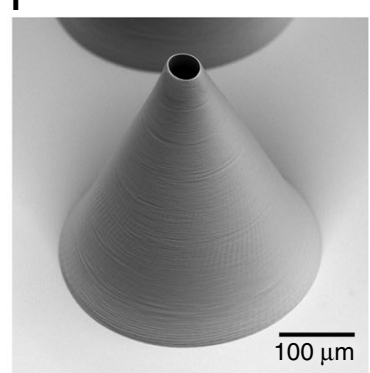

Fig. 1 Overview of fabricated microneedle designs. CAD design $\mathbf{a}$ and SEM images showing a side view $\mathbf{b}$ and a top view $\mathbf{c}$ of a pyramid-shaped microneedle with offset bores and design $\mathbf{d}$ and SEM images showing written thin $\mathbf{e}$ and thick $\mathbf{f}$ truncated cone-shaped microneedles

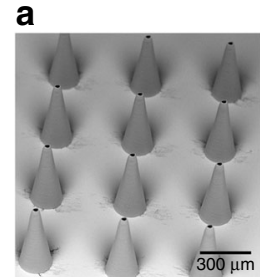

b

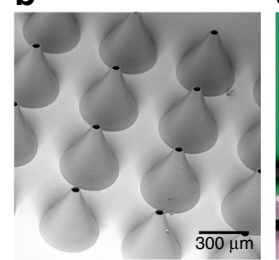

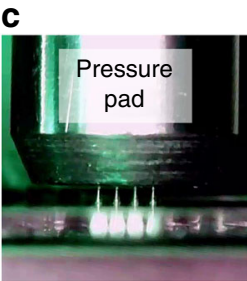

d

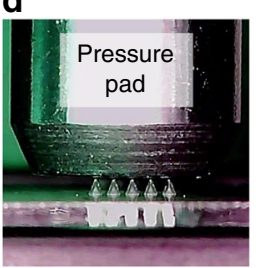

e

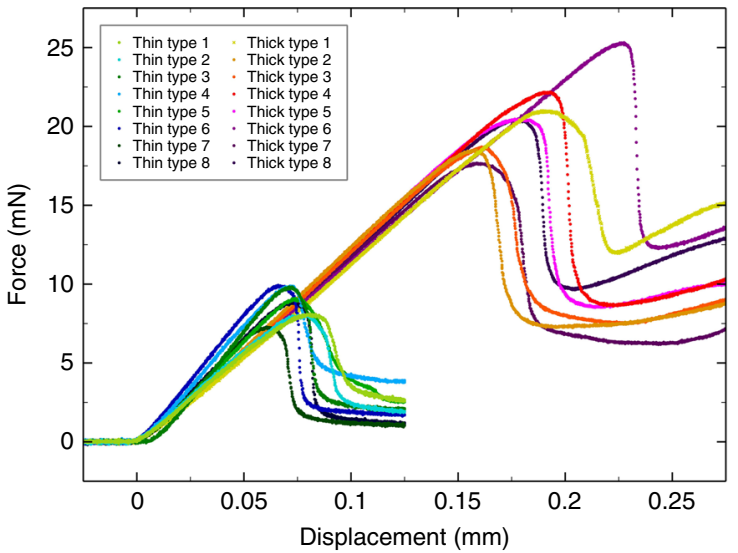

Fig. 2 Compression tests. SEM images showing a detailed view of a thin a type microneedle array and a thick $\mathbf{b}$ type microneedle array, the test setup with a pressure pad lowering down onto thin $\mathbf{c}$ and thick $\mathbf{d}$ microneedle arrays, and $\mathbf{e}$ results per needle of the compression test

deformation. The supplementary videos S1 and S2 also show that the microneedles straighten up again after compression.

In a second compression test, arrays of thin and thick microneedles are fixed to a pressure pad, dyed with rhodamine B solution, and pressed against pork skin surfaces as illustrated in Fig. 3a. After the test, the pork skin is marked in the places where the microneedles interacted with the skin. The impression of a $5 \times 5$ array of thick microneedles is presented in Fig. $3 \mathrm{~b}$ assuming that the middle microneedle in the first row was not sufficiently dyed. In addition, residues of pink rhodamine B solution transferred from the coverslip to the pork skin are visible surrounding the impression of the microneedle array. The microneedle specimens are tested against pork skin several times to ensure that the microneedles maintain their structural integrity after application. For penetration and permeation studies of skin, pork skin is often used as it exhibits a similar morphology as compared to human $\operatorname{skin}^{36}$. Figure $3 \mathrm{c}$ shows the results of the measured force of an array of thick 
a

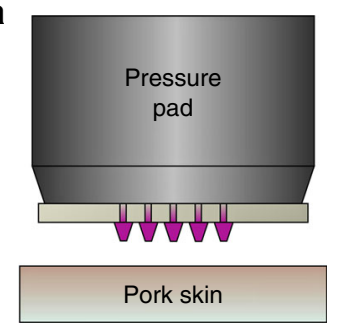

b

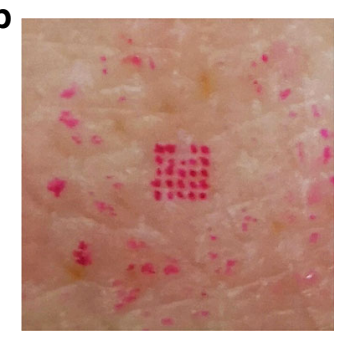

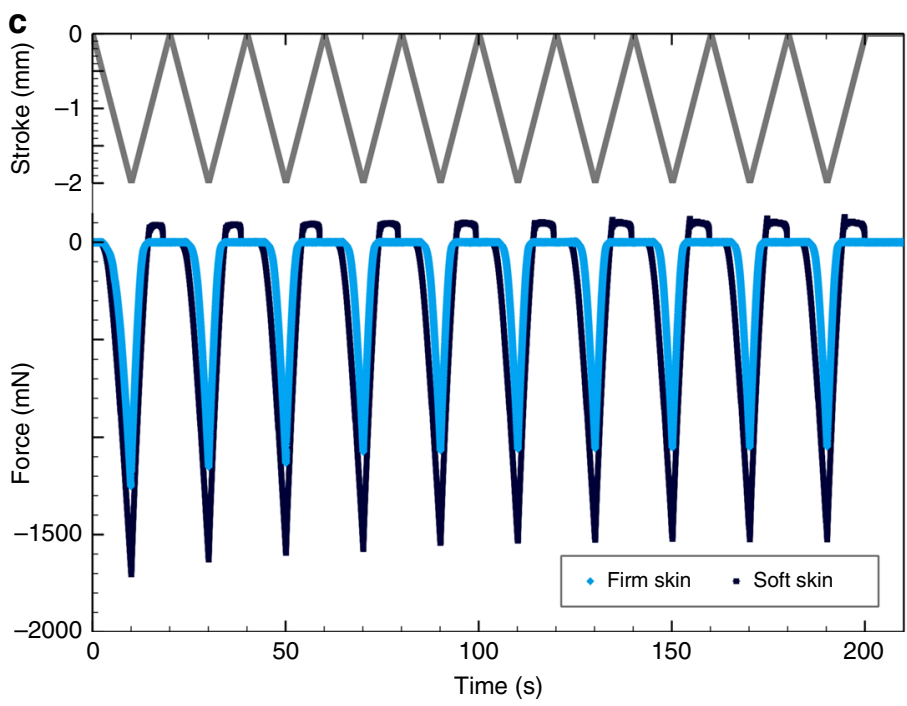

Fig. 3 Microneedles tested against pork skin. Illustration of the test setup $\mathbf{a}$, the impression of an array of thick microneedles onto pork skin $\mathbf{b}$, and results of the measured force of an array of thick microneedles pressed against soft and firm pork skin, in each case performing ten iterations $\mathbf{c}$

microneedles pressed against soft (supplementary video S3) and firm (supplementary video S4) pork skin, in each case performing 10 iterations. The movement of the prepared pressure pad starts above the pork skin surface lowering down onto the skin with a downstroke of $2 \mathrm{~mm}$. The curves show a constant force until the microneedles reach the pork skin surface after a distance of about $1 \mathrm{~mm}$. Then, the curves decrease linearly due to the increasing reaction force of the skin until the turning point is reached. During the upstroke of $2 \mathrm{~mm}$, the curves, in turn, increase linearly. Furthermore, there is an overshoot of the measured force observable for soft skin because the skin adheres to the coverslip. It is worth noting that the first minimum is deeper than the later ones pointing to an additional initial skin penetration force. After the compression tests, the microneedles are still intact.

\section{Flow test}

The success of combining microneedles and microchannels using femtosecond laser-induced nonlinear processes is proved by a flow test. A fluidic system of both, needles generated by direct laser writing and channels created in a two-step process of irradiation and subsequent annealing, is shown in Fig. 4a. Four microneedles connected to a subjacent horizontal channel by vertical channels are clearly visible. The functionality of the microfluidic channels is highlighted in Fig. 4b presenting a branched network filled with a rhodamine B/distilled water solution. To ensure that liquids with different viscosity are applicable with the microneedle-based system, we successfully tested the throughput of rhodamine $\mathrm{B} /$ distilled water solution with a viscosity of $\sim 1 \mathrm{mPa} \cdot \mathrm{s}$ and of ethylene glycol with a viscosity of $20.81 \mathrm{mPa} \cdot \mathrm{s}$ at $20^{\circ} \mathrm{C}$. Both liquids run through the openings of the vertical channels with a diameter of $30 \mu \mathrm{m}$, which is similar to the tip diameter of the truncated cone-shaped microneedles. Moreover, the rhodamine $\mathrm{B} /$ distilled water solution is injected through the opening for fluid supply into a combined system and, as expected, the solution leaks from the microneedles. A microneedle with residues of pink rhodamine B solution from the flow test is shown in Fig. 4c. This proof of principle microfluidic demonstration underlines the potential of such a microfluidic system for fluid injection and extraction.

\section{Parameter study}

Microneedles as well as microfluidic channels are generated with $515 \mathrm{~nm}$ ultrashort pulse lasers. The processes so far only differ on repetition rate and pulse duration assuming that the laser power is adjustable. Other groups $^{37-39}$ have shown that a variation of these two parameters is possible for two-photon polymerization. These results suggest that the repetition rate and pulse duration is adaptable for the polymerization of microneedles towards equal parameters for both laser processes. Taking account of the results of Fischer et al. ${ }^{37}$ for repetition rates above $100 \mathrm{kHz}$, a comparable polymerization behavior can be expected for different laser parameter settings $a$ and $b$ when

$$
P_{a}^{n} \tau_{a} f_{\text {rep }}=P_{b}^{n} \tau_{b} f_{\text {rep }}
$$

where $P_{a}$ and $P_{b}$ are the pulse powers, $\tau_{a}$ and $\tau_{b}$ are the pulse durations, $f_{\text {rep }}$ and $f_{\text {rep }}$ are the repetition rates of 
a

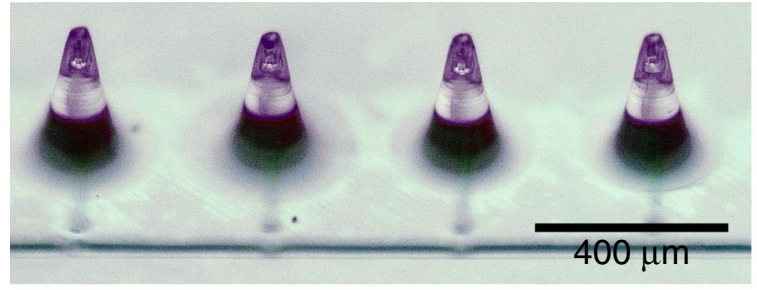

b

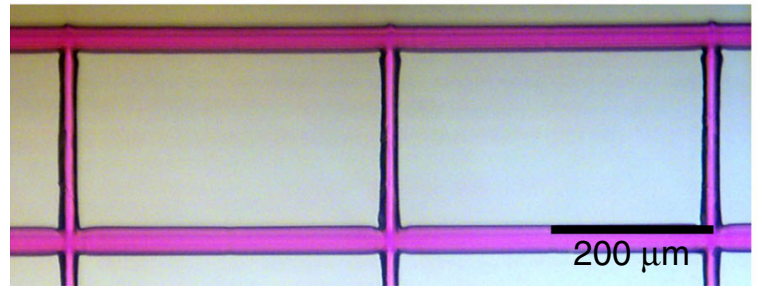

C

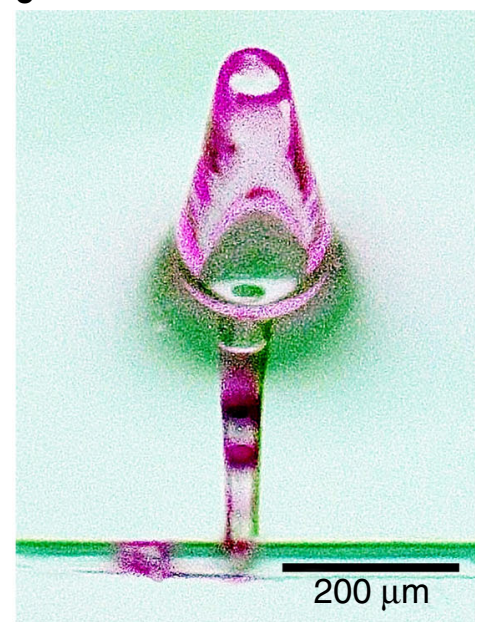

Fig. 4 Flow test. A fluidic system combining microneedles and microchannels $\mathbf{a}$, solution-filled microfluidic channel $\mathbf{b}$, and microneedle with residues of pink rhodamine $B$ solution $\mathbf{c}$

the laser parameter settings and $n$ defines the nonlinear absorption order. Considering this equation, the nonlinear absorption order $n$ of OrmoComp is determined to be two for a wavelength of $515 \mathrm{~nm}$ in a repetition rate range from $100 \mathrm{kHz}$ to $10 \mathrm{MHz}$ keeping the pulse duration constant by $540 \mathrm{fs}$ and in a pulse duration range from $345 \mathrm{fs}$ to $2.8 \mathrm{ps}$ by a constant repetition rate of $10 \mathrm{MHz}$. For this examination, pulse powers were identified to fabricate a three-dimensional scaffold in identical quality varying the repetition rate and pulse duration using a writing speed of $1 \mathrm{~mm} / \mathrm{s}$. Figure $5 \mathrm{a}$ presents a part of one of the matrices used for the power determination writing scaffolds with increasing repetition rates from bottom to top and increasing power from left to right. There seems to be no influence of the repetition rate or pulse duration on the structuring quality for the investigated laser parameters. It is expected that the repetition rates and pulse durations are in a parameter window ${ }^{39}$, which allows an accumulation of the exposure $\operatorname{doses}^{37}$ of the single laser pulse in the exposure window.

Furthermore, Eq. (1) allows the calculation of a pulse power $P_{b}$ for successful direct laser writing with a pulse duration $\mathrm{\tau}_{b}$ and repetition rate $f_{\text {rep }}$ when an appropriate laser power $P_{a}$ exists for direct laser writing with a pulse duration $\tau_{a}$ and repetition rate $f_{\text {rep }}$, the nonlinear absorption order $n$ is known and all other parameters are equal. A laser power range to fabricate microneedles using a repetition rate of $10 \mathrm{MHz}$ and a pulse duration of $540 \mathrm{fs}$ is determined, and power ranges for other repetition rates and pulse durations are calculated on the basis of this result. According to Eq. (1), the calculated ranges are limited by two straight lines in a double logarithmic plot as presented with dashed lines in Fig. 5. Additionally, an overview of the selected and successfully tested values for writing microneedles is given in Fig. 5. This method for predicting an adequate laser power is extended to a repetition rate of $61 \mathrm{kHz}$ and a pulse duration of $450 \mathrm{fs}$ enabling the polymerization of microneedles with the same parameters and laser setup used for the microchannel generation. Figure $5 \mathrm{c}$ shows the power values suitable for microneedle fabrication under these conditions.

\section{Discussion}

The combination of microneedles and microchannels as presented in this work allows the fabrication of versatile drug injection and fluid extraction systems with only a few processing steps. This is an advantage over laborious multiple processing methods ${ }^{7,27-29}$. It is even possible to generate both, microneedles and microchannels, using the same laser system as demonstrated in the previous section, providing the technological basis of a true hybrid process.

An important point is the penetration ability of the needles into human skin. Doraiswamy et al ${ }^{40}$ performed first experiments testing OrmoComp microneedles against pork skin. After interaction with the skin, intact microneedles without fracture were observed. Moreover, Davis et al. ${ }^{35}$ present a comprehensive investigation of the insertion of microneedles into human skin. For microneedles with a tip radius of $30-80 \mu \mathrm{m}$, insertion forces from 0.08 to $3.04 \mathrm{~N}$ were measured. The attendant diagram $^{35}$ indicates that the insertion force for our microneedles with a tip radius of $13 \pm 2 \mu \mathrm{m}$ (thin needles) and $21 \pm 2 \mu \mathrm{m}$ (tick needles) is expected in a range of $5 \mathrm{mN}$. This is less than the maximal force $(7.3-9.9 \mathrm{mN}$ for thin needles and $17.7-25.3 \mathrm{mN}$ for thick needles) measured during the first compression test. Roxhed et al. ${ }^{41}$ designed 

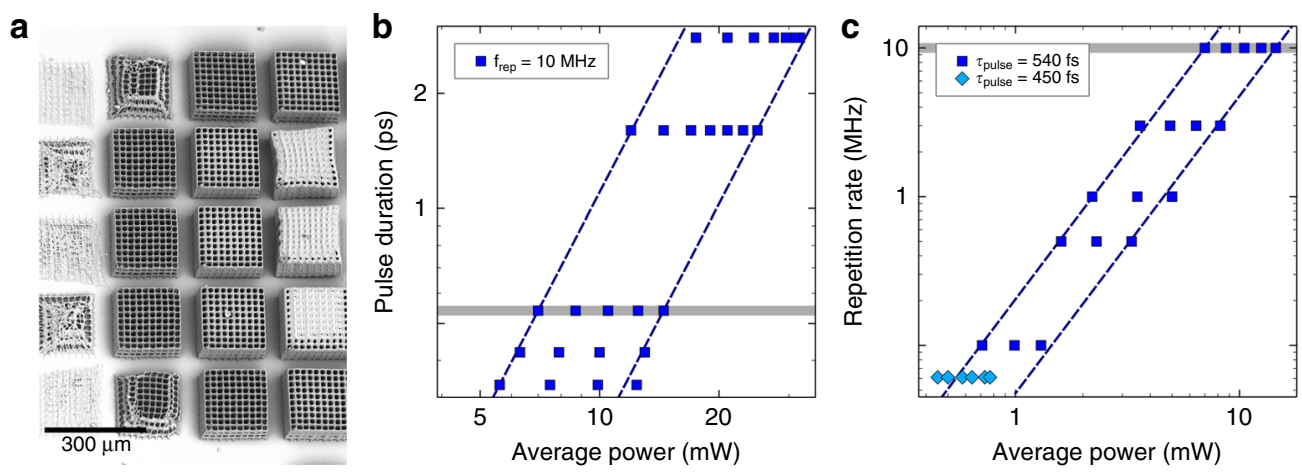

Fig. 5 Parameter study. Three-dimensional objects with bottom-up increasing repetition rate and rightwards increasing pulse powers a and average laser powers for laser direct writing of microneedles with different pulse durations $\mathbf{b}$ and repetition rates $\mathbf{c}$, whereby dashed lines show calculated power ranges based on the results for $10 \mathrm{MHz}$ and $540 \mathrm{fs}$ and points present successfully tested values

microneedles with a sharp tip and the insertion force of a circular microneedle type was estimated to be below 10 $\mathrm{mN}$. This example clearly shows that an insertion force below $10 \mathrm{mN}$ is sufficient to penetrate the skin and that pyramid-shaped microneedles with a sharp tip, as presented in Fig. 1, are of interest. Altogether, laser direct written microneedles made from OrmoComp are a good choice for further microneedle-based experiments due to their stability, biocompatibility, and the ability to realize arbitrarily shaped needles.

\section{Materials and methods Microchannel generation}

Microchannels are created in PMMA substrate material by a laser-induced process consisting of two process steps, outlined in Fig. 6. First, the specimens are irradiated by focused femtosecond pulses in a multi-pass process triggering a nonlinear absorption inside the focal volume. The presented process to generate hollow structures inside PMMA is based on a multi-pass scanning approach to achieve a higher microchannel quality. As also observed by Rekštyte et al. $^{42}$, thermal accumulation, which can be attributed to a too small pulse to pulse distance, has an impact on the results of the two-photon polymerization process. We prevent this thermal accumulation by using a high scanning speed of $20 \mathrm{~mm} / \mathrm{s}$. In combination with a limited peak intensity by nonlinear beam collapsing, the multi-scanning approach is a possibility to achieve a sufficient high irradiation dose for an internal modification of PMMA. Definable 3D geometries can be created by moving the focal spot in three dimensions inside the bulk. As shown by Baum et al. ${ }^{43}$, femtosecond laser-irradiated PMMA exhibits a lower thermal stability as compared to pristine material. After laser processing, the specimens are placed on a hot plate between two glass substrate and are annealed at $200^{\circ} \mathrm{C}$ for $30 \mathrm{~s}$. Modified material is degenerated and internal microchannels are formed. It is worth noting that prior to annealing no microchannels are found, i.e., their formation is clearly linked to the annealing process. Details on this process can be found in Roth et al. ${ }^{31}$.

In this study, laser processing is performed using an Ytterbium-doped Potassium Gadolinium Tungstate (Yb:KGW) ultrashort pulse laser (Light Conversion, Pharos-10-600) with a wavelength of $515 \mathrm{~nm}$, a pulse duration of $450 \mathrm{fs}$, and a pulse repetition rate of $61 \mathrm{kHz}$. The laser is focused by a $20 \mathrm{x}$ objective with an NA of 0.5 (Zeiss, EC Epiplan-Neofluar) movable along the laser beam direction by a nanopositioning $z$-stage (Aerotech, ANT95-50-L-Z). Samples are translated using a linear stage (Aerotech, ANT130-XY). Horizontal microchannels are generated at a writing speed of $20 \mathrm{~mm} / \mathrm{s}$ and vertical microchannels at a writing speed of $10 \mathrm{~mm} / \mathrm{s}$.

\section{Microneedle fabrication}

The microneedles are mainly written with a laser system designed for two-photon polymerization. An Ytterbiumdoped femtosecond oscillator (Amplitude Systems, Mikan) having a pulse duration of $300 \mathrm{fs}$ and a repetition rate of $55 \mathrm{MHz}$ at $515 \mathrm{~nm}$ is used to realize arbitrarily shaped microneedles designed with a CAD software. For further studies, a BlueCut femtosecond fiber laser (Menlo Systems) extended by a second-harmonic generation setup was integrated. This laser system provides light pulses at a wavelength of $515 \mathrm{~nm}$ with adjustable pulse duration from 345 fs to $2.8 \mathrm{ps}$ and variable repetition rate from $100 \mathrm{kHz}$ up to $10 \mathrm{MHz}$. For direct writing, the expanded laser beam from one of the two lasers is focused by a 20x objective with an NA of 0.5 (Zeiss, EC EpiplanNeofluar) or, alternatively, a 63x objective with an NA of 0.75 (Zeiss, LD Plan-Neofluar) into a photosensitive material layer consisting of OrmoComp (micro resist technology). 


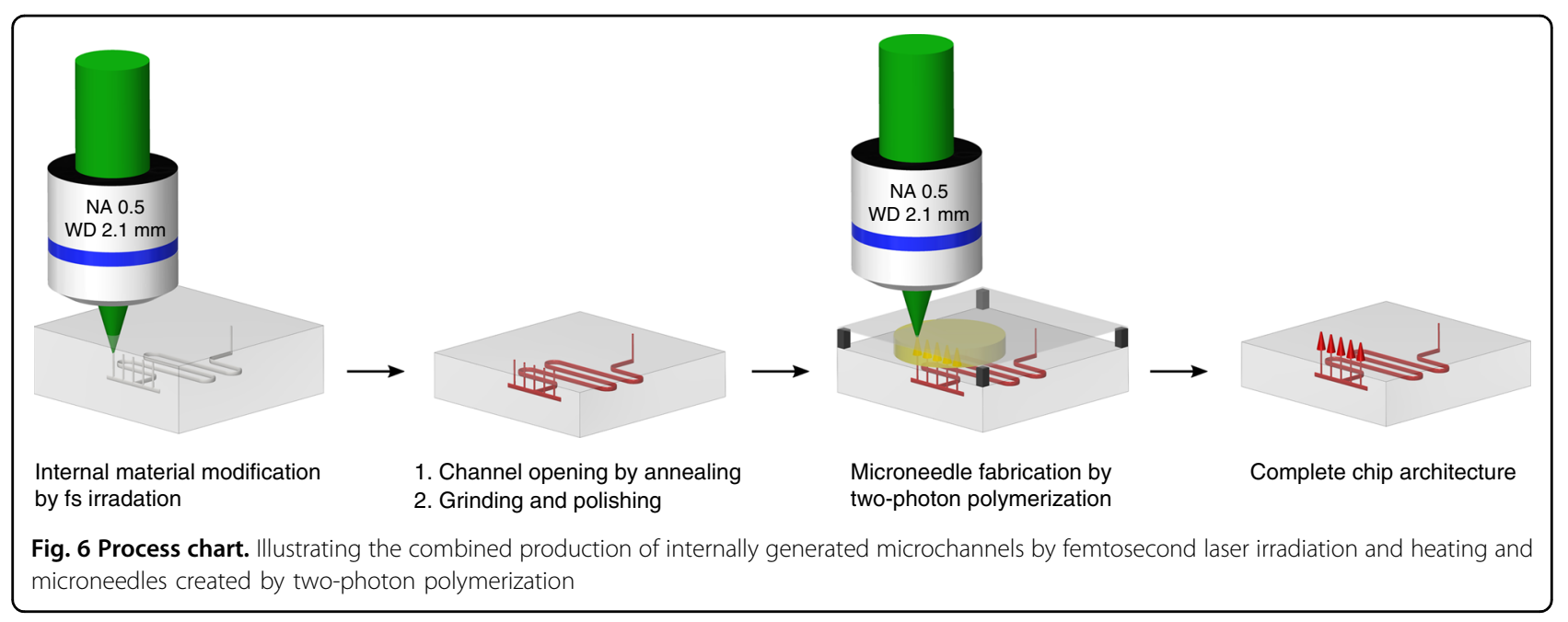

Microneedles are built up on a subjacent substrate, glass coverslip with laser-drilled holes, or prepared PMMA chip with microchannels as presented in Fig. 6 (3). A flat surface is achieved by a cover glass above the photosensitive material layer and avoids additional aberration. The samples are placed on a plate, which is movable in the plane perpendicular to the incoming beam using a translation stage (Aerotech, ANT130-XY). For real threedimensional structures, the objective is movable along the laser beam direction by a nanopositioning $z$-stage (Aerotech, ANT95-50-L-Z). An integrated CCD camera qualifies to identify the holes and openings of the vertical microchannels in the substrate and to position the designed microneedles correctly onto these openings. The entire process combining direct laser written microneedle arrays and femtosecond laser generated microchannels is shown in Fig. 6.

\section{Mechanical compression test of microneedle arrays}

Two different types of truncated cone-shaped microneedle arrays are written on glass coverslips with laserdrilled holes using a Mikan laser system and analyzed by mechanical compression tests. $4 \times 4$ arrays of thin microneedles (with a base radius of $100 \mu \mathrm{m}$, a tip radius of $15 \mu \mathrm{m}$, a height of $300 \mu \mathrm{m}$, and 500 turns) and $5 \times 5$ arrays of thick microneedles (with a base radius of $187.5 \mu \mathrm{m}$, a tip radius of $20 \mu \mathrm{m}$, a height of $250 \mu \mathrm{m}$, and 500 turns) are fabricated applying the $63 \mathrm{x}$ objective, a laser power of $13.5 \mathrm{~mW}$, and a writing speed of $5 \mathrm{~mm} / \mathrm{s}$. This exposure of the polymer OrmoComp is followed by a post-baking for $10 \mathrm{~min}$ at $130^{\circ} \mathrm{C}$, developing in OrmoDev (micro resist technology) for $60 \mathrm{~min}$, and hard baking for $3 \mathrm{~h}$ at $150^{\circ} \mathrm{C}$. One after the other, the samples are attached to the base plate of a UMT TriboLab system (Bruker), and a pressure pad with a flat surface goes down to the microneedle array at a constant speed of $0.015 \mathrm{~mm} / \mathrm{s}$. For each sample, force and displacement of the pressure pad are measured and recorded during compression.

\section{Microfluidic demonstration}

In order to combine microneedles and microchannels, PMMA chips with an internal three-dimensional microchannel architecture are ground down to open the vertical channels. After this process step, the specimens are rinsed in an ultrasonic bath for $10 \mathrm{~min}$ to clean the microchannels. Before OrmoComp is applied to the samples for the two-photon polymerization process, additional openings for fluid supply, which are not covered with the photosensitive material, are temporarily closed to reduce the ingress of the polymer into the channels. A laser power of $12 \mathrm{~mW}$, a repetition rate of $10 \mathrm{MHz}$, a pulse duration of $540 \mathrm{fs}$, a writing speed of $5 \mathrm{~mm} / \mathrm{s}$, and the $63 \mathrm{x}$ objective are appropriate to place microneedles with a base radius of $175 \mu \mathrm{m}$, a tip radius of $25 \mu \mathrm{m}$, a height of $400 \mu \mathrm{m}$, and 400 turns onto the channel openings. The non-polymerized material is carefully removed with distilled water to protect the PMMA chip against being damaged by OrmoDev or isopropanol. A rhodamine B/ distilled water solution is injected inside the rinsed microfluidic system through the openings for fluid supply. This is observed and pictures are taken by a swingable digital microscope (Leica, DVM6).

\section{Variation of pulse duration and repetition rate}

The extended BlueCut laser system is variable in pulse duration and repetition rate. Thus, it enables a closer examination of these parameters regarding two-photon polymerization. First, an adequate laser power range is identified to fabricate microneedles with a repetition rate of $10 \mathrm{MHz}$ and a laser pulse duration of $540 \mathrm{fs}$ using the $20 \mathrm{x}$ objective and a writing speed of $5 \mathrm{~mm} / \mathrm{s}$. The result serves as a starting point for the evaluation of other repetition rate and pulse duration values. The repetition 
rate is gradually reduced choosing $10,3,1 \mathrm{MHz}, 500$, and $100 \mathrm{kHz}$ with a constant pulse duration of $540 \mathrm{fs}$. Additionally, the pulse duration is varied from 345 fs to $2.8 \mathrm{ps}$ adjusting $345,420,540 \mathrm{fs}, 1.6$, and 2.8 ps by means of an autocorrelator (pulseCheck, APE) whereby the repetition rate is kept constant at $10 \mathrm{MHz}$. The average laser powers are measured behind the objective with an incoming beam diameter of $7.7 \mathrm{~mm}$ measured at $1 / \mathrm{e}^{2}$.

\section{Conclusion}

In this study, a microfluidic system of direct laser written microneedles combined with internal laser generated microchannels is realized by a hybrid approach. In particular, reproducible microneedles are exactly positioned on openings of vertical microchannels, which build a branched network with horizontal channels. A successful microfluidic examination with rhodamine B solution demonstrates the applicability of such a system for fluid injection and extraction. Furthermore, the results of a compression analysis suggest that laser direct written microneedles made from OrmoComp ${ }^{\oplus}$ are a promising candidate for medical microneedle applications. We identified that only a single laser system is required for both processes defining a true hybrid femtosecond laser process. This is a significant advance compared to other fabrication methods requiring several systems for multiple processing steps creating a microfluidic system with microneedles. In summary, the achieved results are a fundamental and promising basis for further research towards a versatile point-of-care system combining femtosecond laser generated microfluidic channels and direct laser written microneedle arrays.

\section{Acknowledgements}

This work was supported by the European program "Eurostars" under the project number E!9765 (Hybrid-3D) and funded by the German partner with resources from the German Federal Ministry of Education and Research (BMBF).

\section{Author contributions}

A.T. performed the flow tests, the parameter study, and wrote part of this manuscript. G.-L. R. performed the microchannel generation and wrote part of this manuscript. B.N. performed the mechanical compression test. T.W. and R.H. supervised this project and supported the writing of this manuscript.

\section{Conflict of interest}

The authors declare that they have no conflict of interest.

Supplementary information accompanies this paper at https://doi.org/ 10.1038/s41378-019-0046-5.

Received: 23 August 2018 Revised: 14 December 2018 Accepted: 3 January 2019

Published online: 25 February 2019

\footnotetext{
References

1. Xie, S., Li, Z. \& Yu, Z. Microneedles for transdermal delivery of insulin. J. Drug Del. Sci. Tech. 28, 11-17 (2015).
}

2. Donnelly, R. \& Larrañeta, E. Microarray patches: potentially useful delivery systems for long-acting nanosuspensions. Drug Discov. Today 23, 1026-1033 (2018).

3. Kim, Y., Park, J. \& Prausnitz, M. Microneedles for drug and vaccine delivery. Adv. Drug Deliv. Rev. 64, 1547-1568 (2012).

4. Maaden, $\mathrm{K}$. et al. Hollow microneedle-mediated micro-injections of a liposomal HPV E74363 synthetic long peptide vaccine for efficient induction of cytotoxic and t-helper responses. J. Control. Release 269, 347-354 (2018).

5. Caffarel-Salvador, E. et al. Potential of hydrogel-forming and dissolving microneedles for use in paediatric populations. Int. J. Pharm. 489, 158-169 (2015).

6. Mukerjee, E., Collins, S., Isseroff, R. \& Smith, R. Microneedle array for transdermal biological fluid extraction and in situ analysis. Sens. Actuators A 114, 267-275 (2004).

7. Miller, P. et al. Towards an integrated microneedle total analysis chip for protein detection. Electroanalysis 28, 1147-1400 (2016).

8. Larraneta, E., Lutton, R., Woolfson, A. \& Donnelly, R. Microneedle arrays as transdermal and intradermal drug delivery systems: materials science, manufacture and commercial development. Mater. Sci. Eng. 104, 1-32 (2016).

9. Shah, U. et al. Needle-free and microneedle drug delivery in children: a case for disease-modifying antirheumatic drugs (dmards). Int. J. Pharm. 416, 1-11 (2011).

10. Gupta, J., Park, S., Bondy, B., Felner, E. \& Prausnitz, M. Infusion pressure and pain during microneedle injection into skin of human subjects. Biomaterials 32, 6823-6831 (2011)

11. Lim, D., Vines, J., Park, H. \& Lee, S. Microneedles: a versatile strategy for transdermal delivery of biological molecules. Int. J. Biol. Macromol. 110, 30-38 (2018).

12. Ita, K. Transdermal delivery of drugs with microneedles: strategies and outcomes. J. Drug Deliv. Sci. Tech. 29, 16-23 (2015).

13. Bal, S., Caussin, J., Pavel, S. \& Bouwstra, J. In vivo assessment of safety of microneedle arrays in human skin. Eur. J. Pharm. Sci. 35, 193-202 (2008).

14. Gill, H. \& Prausnitz, M. Coated microneedles for transdermal delivery. J. Control. Release 117, 227-237 (2007).

15. Ling, M. \& Chen, M. Dissolving polymer microneedle patches for rapid and efficient transdermal delivery of insulin to diabetic rats. Acta Biomater. $\mathbf{9}$, 8952-8961 (2013).

16. Zhu, D., Wang, Q., Liu, X. \& Guo, X. Rapidly separating microneedles for transdermal drug delivery. Acta Biomater. 41, 312-319 (2016).

17. Doraiswamy, A. et al. Fabrication of microneedles using two photon polymerization for transdermal delivery of nanomaterials. J. Nanosci. Nanotechnol. 10, 6305-6312 (2010)

18. $\mathrm{Ma}, \mathrm{G} . \& \mathrm{Wu}, \mathrm{C}$. Microneedle, bio-microneedle and bio-inspired microneedle: a review. J. Control. Release 251, 11-23 (2017).

19. Miller, P. et al. Electrodeposited iron as a biocompatible material for microneedle fabrication. Electroanalysis 27, 2239-2249 (2015).

20. Kong, X. \& Wu, C. Measurement and prediction of insertion force for the mosquito fascicle penetrating into human skin. J. Bionic. Eng. 6, 143-152 (2009).

21. Izumi, H., Suzuki, M., Aoyagi, S. \& Kanzaki, T. Realistic imitation of mosquito's proboscis: electrochemically etched sharp and jagged needles and their cooperative inserting motion. Sens. Actuators A 165, 115-123 (2011).

22. Ashraf, M. et al. Design simulation and fabrication of silicon microneedles for bio-medical applications. ECTI-CON 9, 83-91 (2010).

23. Sammoura, F., Kang, J., Heo, Y., Jung, T. \& Lin, L. Polymeric microneedle fabrication using a microinjection molding technique. Microsyst. Technol. 13, 517-522 (2007).

24. Gittard, S. et al. The effects of geometry on skin penetration and failure of polymer microneedles. J. Adhes. Sci. Technol. 27, 227-243 (2013).

25. Marino, A. et al. The osteoprint: a bioinspired two-photon polymerized 3-d structure for the enhancement of bone-like cell differentiation. Acta Biomater. 10, 4304-4313 (2014)

26. Trautmann, A., Rüth, M., Lemke, H., Walther, T. \& Hellmann, R. Two-photon polymerization based large scaffolds for adhesion and proliferation studies of human primary fibroblasts. Opt. Laser Technol. 106, 474-480 (2018).

27. Wang, H., Pastorin, G. \& Lee, C. Toward self-powered wearable adhesive skin patch with bendable microneedle array for transdermal drug delivery. Adv. Sci. 3, 1500441 (2016).

28. Paik, S. et al. In-plane single-crystal-silicon microneedles for minimally invasive microfluid systems. Sens. Actuators A 114, 276-284 (2004). 
29. Kim, B. et al. Touch-actuated transdermal delivery patch for quantitative skin permeation control. Sens. Actuators B 256, 18-26 (2018).

30. Rad, Z. et al. High-fidelity replication of thermoplastic microneedles with open microfluidic channels. Microsyst. Nanoeng. 3, 17034 (2017).

31. Roth, G., Esen, C. \& Hellmann, R. Femtosecond laser direct generation of 3Dmicrofluidic channels inside bulk PMMA. Opt. Express 25, 18442-18450 (2017)

32. Jonušauskas, L. et al. Hybrid subtractive-additive-welding microfabrication for lab-on-chip applications via single amplified femtosecond laser source. Opt. Eng. 56, 094108 (2017).

33. Tičkūnas et al. Combination of additive and subtractive laser 3D microprocessing in hybrid glass/polymer microsystems for chemical sensing applications. Opt. Express 25, 26280-26288 (2017).

34. Tsao, C. Polymer microfluidics: simple, low-cost fabrication process bridging academic lab research to commercialized production. Micromachines 7, 225 (2016).

35. Davis, S., Landis, B., Adams, Z., Allen, M. \& Prausnitz, M. Insertion of microneedles into skin: measurement and prediction of insertion force and needle fracture force. J. Biomech. 37, 1155-1163 (2004).

36. Diembeck, W. et al. Test guidelines for in vitro assessment of dermal absorption and percutaneous penetration of cosmetic ingredients. Food Chem. Toxicol. 37, 191-205 (1999).
37. Fischer, J. et al. Three-dimensional multi-photon direct laser writing with variable repetition rate. Opt. Express 21, 26244-26260 (2013).

38. Malinauskas, M., Danilevicius, P. \& Juodkazis, S. Three-dimensional micro-/ nano-structuring via direct write polymerization with picosecond laser pulses. Opt. Express 19, 5602-5610 (2011).

39. Tomova, Z., Liaros, N., Razo, S., Wolf, S. \& Fourkas, J. In situ measurement of the effective nonlinear absorption order in multiphoton photoresists. Laser Photonics Rev. 10, 849-854 (2016).

40. Doraiswamy, A. et al. Two photon induced polymerization of organicinorganic hybrid biomaterials for microstructured medical devices. Acta Biomater. 2, 267-275 (2006)

41. Roxhed, N., Gasser, T., Griss, P., Holzapfel, G. \& Stemme, G. Penetrationenhanced ultrasharp microneedles and prediction on skin interaction for efficient transdermal drug delivery. J. Micro. Syst. 16, 1429-1440 (2007).

42. Rekštytè, S., Malinauskas, M. \& Juodkazis, S. Three-dimensional laser microsculpturing of silicone: towards bio-compatible scaffolds. Opt. Express 21, 17028-17041 (2013)

43. Baum, A., Scully, P. J., Perrie, D., Liu, D. \& Lucarini, V. Mechanisms of femtosecond laserinduced refractive index modification of poly (methyl methacrylate). J. Opt. Soc. Am. B 27, 107-111 (2010). 